Die Zellen wurden mit kontinuierlicher Intensität zwischen vier 200-Watt-Metallfadenlampen, die nahe an den Kulturkolben herangebracht wurden, gezüchtet. Einsaat $15 \mathrm{~mm}^{3}$ Zellen in $250 \mathrm{~cm}^{3}$ Kulturlösung $\mathrm{K}$, Ernte nach $24 \mathrm{Stdn} .178 \mathrm{~mm}^{3}$. $p_{\mathrm{H}}$ war anfangs 4,3 und bei der Ernte 5,2. Die Zellen wurden sorgfältig 3-mal in Salzlösung $S$
$\left(p_{\mathrm{H}} 3,8\right)$ gewaschen, um alles Bicarbonat zu entfernen. Man beachte, daß bei diesen chlorophyllarmen Zellen die Kontrolle ohne Fluorid sehr viel mehr ausmachte als bei den Zellen des Protokolls 1. Chlorophyllgehalt im Ge$\mathrm{fäß}$ in $160 \mathrm{~mm}^{3}$ Zellen 0,433 $\mu$ Mole. Kegel ohne Einsätze mit 1 Birne. $20^{\circ}$. Dunkel.

\title{
Taxonomie und Cytologie von zwei Subspezies der Art Chironomus thummi
}

\author{
Von H.-G. Keyl und K. Strenzke \\ Aus dem Max-Planck-Institut für Meeresbiologie, \\ Abt. H. B a u e r und Laboratorium K. S tre n z k e, Wilhelmshaven \\ (Z. Naturforschg. 11 b, 7ะ7-735 [1956]; eingegangen am 23. Juli 1956)
}

\begin{abstract}
2 Unterarten von Chironomus thummi unterscheiden sich in ihren Speicheldrüsen-Chromosomen durch eine Anzahl unilokaler Strukturveränderungen, insbesondere den Grad der Anschwellung (Hetero-Chromatinisierung) der Kinetochor-Region. Die morphologischen Unterschiede beziehen sich in erster Linie auf Muster der Körperpigmentierung; Merkmale der Chätotaxie sind variabler. Obwohl die Unterarten im Laboratorium leicht miteinander kreuzbar sind und voll fertile $\mathrm{F}_{1}$-Bastarde liefern, ließ sich experimentell eine ausgeprägte sexuelle Isolation nachweisen. Aus den Freiland-Befunden wird auf eine verschiedene ökologische Einnischung der beiden Unterarten geschlossen.
\end{abstract}

I m Gegensatz zu unseren umfangreichen Kenntnissen über die Artdifferenzierung bei Drosophila ${ }^{1}$ liegen für andere Dipteren nur wenige Untersuchungen über bastardierungs-fähige Arten vor. Aus der Gattung Chironomus sind zwei Fälle bekannt geworden: das Artenpaar Ch. (Camptochironomus) tentans und pallidivittatus durch B e e r m a n n² und ein Formenpaar unbekannten systematischen Ranges von Ch. thummi durch Golds ch m id t ${ }^{3}$.

Durch Zufall fiel uns ein Bastard zweier thummiFormen in die Hand, der große Ähnlichkeit mit dem von G o l d s c h m i d t beschriebenen zu haben schien und zur weiteren Aufklärung cytologisch (Teil II, Verf.: Ke y l) und taxonomisch-ökologisch (Teil I u. III, Verf. S t re n z k e) näher untersucht wurde.

Gelege, die von den Imagines einer aus dem Freiland ins Laboratorium übertragenen Larven-Population von Chironomus thummi $\mathrm{K}$ i e f f. erhalten wurden, erwiesen sich z. T. als nur partiell entwicklungsfähig. Die cytologische Untersuchung durch B a u e ${ }^{*}$ ergab, daß die aus

* Herrn Prof. Dr. H. B a u e r danken wir für die Anregung zu dieser Arbeit.

1 J. T. Patterson u. W. Stone, Evolution in the genus Drosophila, Macmillan, New York 1952.

$2 \mathrm{~W}$. B e e rm a n n, Chromosoma 7, 198 [1955].

3 E. G old s ch midt, J. Heredity 33, 265 [1942].

4 Tendipedidae in ,Die Fliegen der palaearktischen Region“, Stuttgart 1937. diesen Gelegen aufgezogenen Larven ausnahmslos Speicheldrüsen-Chromosomen besaßen, die wie bei den von Goldschmidt bearbeiteten Bastarden streckenweise nicht gepaart waren. Durch Einzelaufzucht vollständig entwickelter Gelege und laufende cytologische Kontrolle konnten die beiden genetisch verschiedenen Sippen isoliert und, nachdem die Milieuansprüche der Larven und Imagines geklärt worden waren, in Laboratoriums-Zuchten gehalten werden. Die Identität der herausgezüchteten Stämme mit den Elternformen der ursprünglich beobachteten heterozygoten Bastardgelege konnte im Kreuzungsversuch bestätigt werden.

\section{I.}

Die beiden reingezüchteten Formen gehören zu Chironomus thummi Kieffer (sensu Goetghebuer ${ }^{4}$;

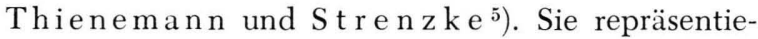
ren Subspezies dieser Art (Chironomus thummi thummi Ki eff. und Ch. th. piger n. subsp. S tr.) ${ }^{*}$,

5 A. Thi en e ma n n u. K. St re n zke, Ent. Tidskr. 72, 1 [1951].

* Die Identifikation der Nominatform mit thummi erfolgt auf Grund der K i e f f e r schen Beschreibung, die mit den von $\mathrm{K}$ i e f f e $\mathrm{r}$ bestimmten Exemplaren in der Sammlung Thienemanns übereinstimmt, und des ökologischen Verhaltens dieser Subspezies. Die Zuordnung von piger zu einer der von $\mathrm{K}$ i e f $\mathrm{f}$ e $\mathrm{r}$ beschriebenen Arten oder "Varietäten“ der thummi-Gruppe war weder an Hand der Diagnosen Ki e f f e r s noch durch Fortsetzung S. 728 
deren Imagines sich auch morphologisch trennen lassen.

Die wesentlichen Färbungsunterschiede gehen aus Abb. 1 hervor. Chironomus thummi thummi ist die dunklere Unterart. Die Mesonotalstreifen sind entweder ein-
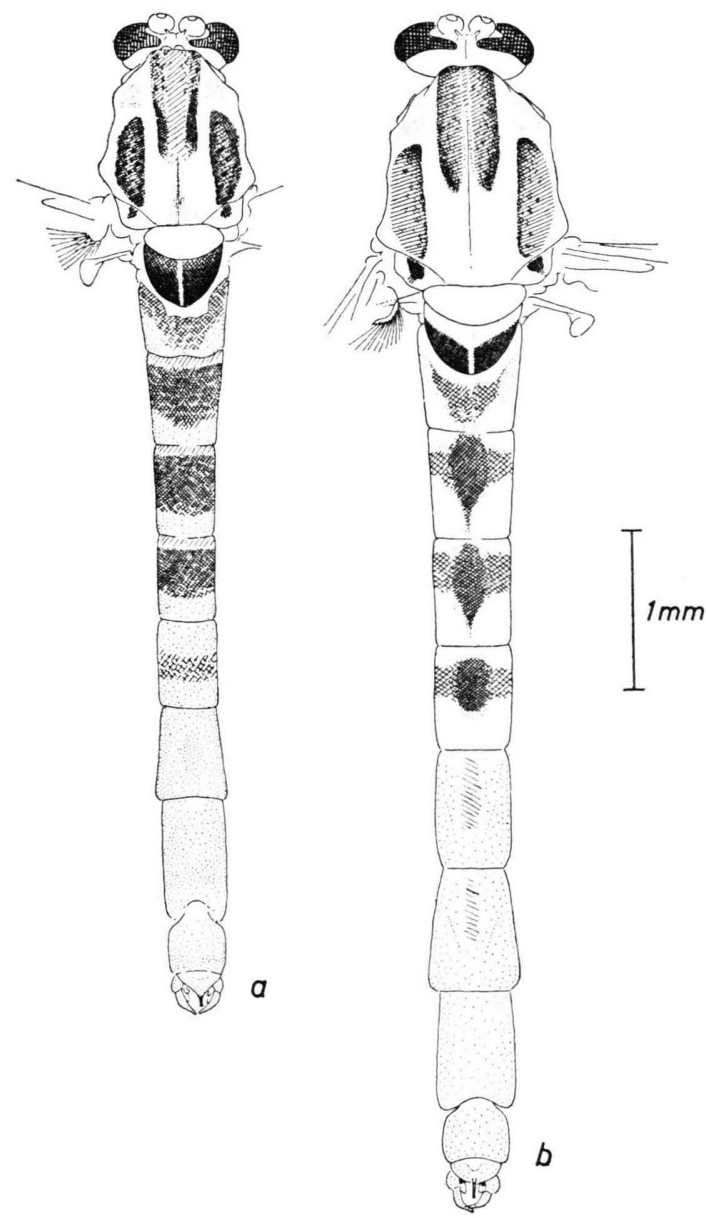

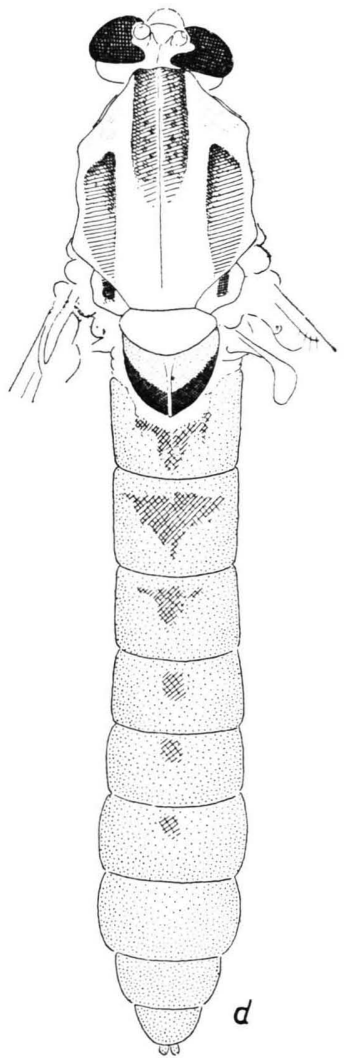

in beiden Geschlechtern gewöhnlich stärker hervor. Die dunkle Zeichnung auf den Abdominaltergiten II und III ist (besonders beim $\hat{\jmath}$ ) meist annähernd rautenförmig; seltener setzt sie in voller Breite dem Oralrand der Tergite an. Mit einem spitz ausgezogenen medianen Fortsatz reicht sie bis oder fast bis zum Hinterrand der Seg-

\section{Abb. 1. Dorsalansicht der Imagines von Chironomus thummi thummi (a fo, c q) und Ch. th. piger (b fo, d + ).}

heitlich schwarzbraun () oder durch schwarzbraune Flecke stark verdunkelt (besonders die Lateralstreifen des 3). Die dunklen Binden auf den Abdominaltergiten II bis IV des $\hat{\jmath}$ können bis ${ }^{4 / 5}$ der Länge des Segments einnehmen; ihre Analränder laufen zu den IntersegmentalHäuten parallel oder sind median nur wenig vorgezogen (Segment II). Bei Chironomus thummi piger tritt die leuchtend rotbraune Grundfärbung der Mesonotalstreifen

Vergleich mit den in der Sammlung Thieneman enthaltenen, von $\mathrm{K}$ i e f f er determinierten Imagines möglich. - Nach mündlicher Mitteilung Herrn Prof. Dobzhanskys besteht neuerdings die Neigung, nur allopatrische taxonomische Einheiten als Subspezies zu bezeichnen. Da es noch offen bleiben muß, wieweit die festgestellte Habitat-Differenzierung der beiden behan- meate. Den klarsten Färbungsunterschied zwischen den beiden Subspezies liefert das Metanotum. Bei thummi ist es, abgesehen von der hellen Medianlinie, einheitlich schwarzbraun, lediglich der Oralteil kann etwas fleckig aufgehellt sein. Bei piger ist dagegen das orale Drittel oder Viertel des Metanotums durch seine hellgelbe ( $\hat{\sigma}$ ) oder rötlich-gelbe (q) Färbung scharf gegen den schwarzbraunen Analteil abgesetzt.

delten Chironomus-Formen durch geographische Momente beeinflußt wird, scheint es, in Übereinstimmung mit dem in der heutigen Chironomiden-Systematik üblichen Verfahren, zweckmäßig, diesen Formen zunächst nur subspezifischen Rang zuzuerkennen. Die definitive Klärung kann erst erfolgen, wenn umfangreiches Material aus verschiedenen Regionen vorliegt. 
H. - G. Keyl und K.Strenzke, Taxonomie und Cytologie von zwei Subspezies der Art Chironomus thummi (S. 727)

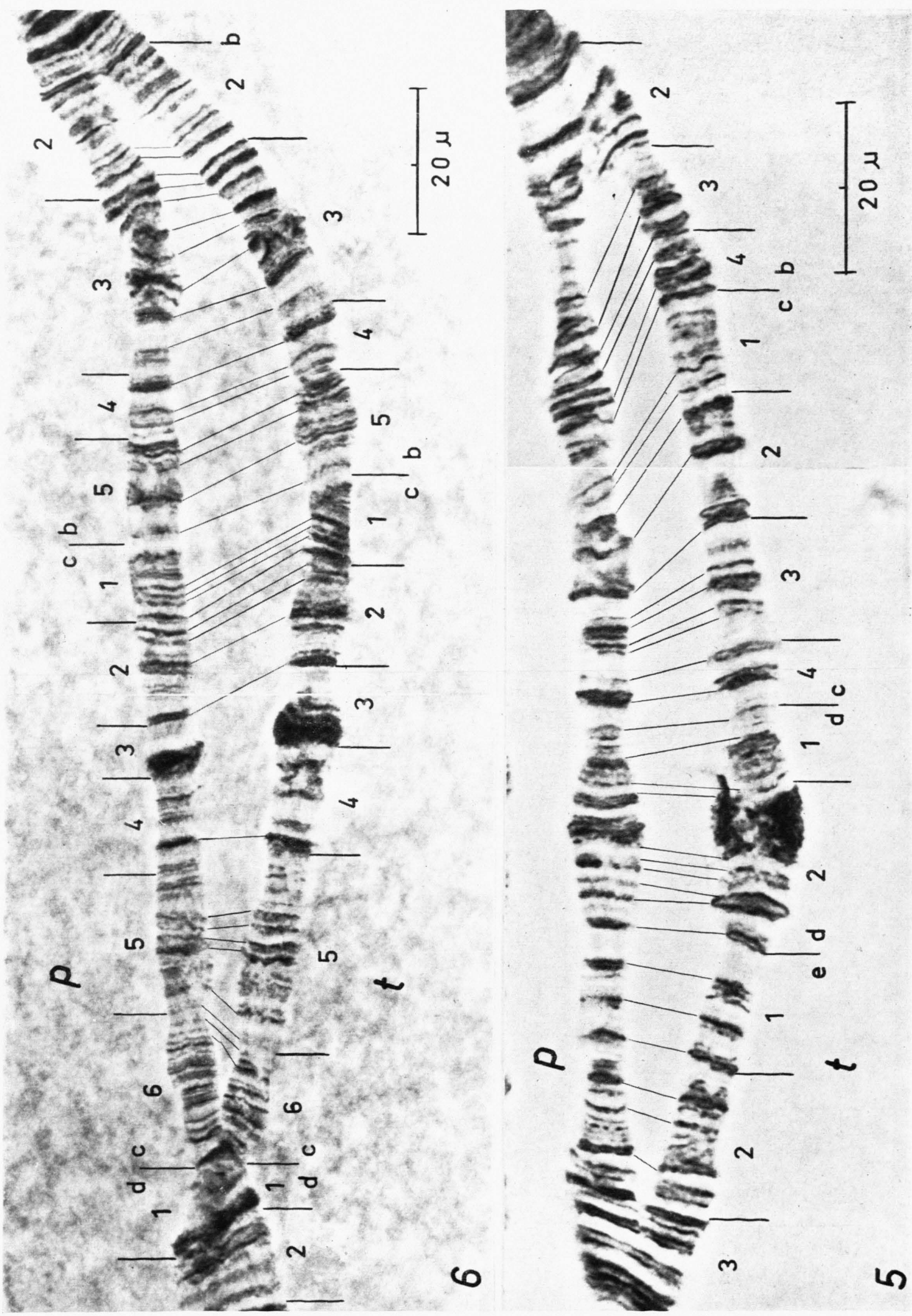

Abb. 5-9. Speicheldrüsen-Chromosomen des Bastards Ch. thummi thummi $(\mathrm{t}) \times$ Ch. th. piger (p). Abb. 5. Heterozygoter Mittelabschnitt von Chromosom I (Vergr. 1400-fach). Abb. 6. Heterozygoter Mittelabschnitt von Chromosom II (Vergr. 1200-fach). 


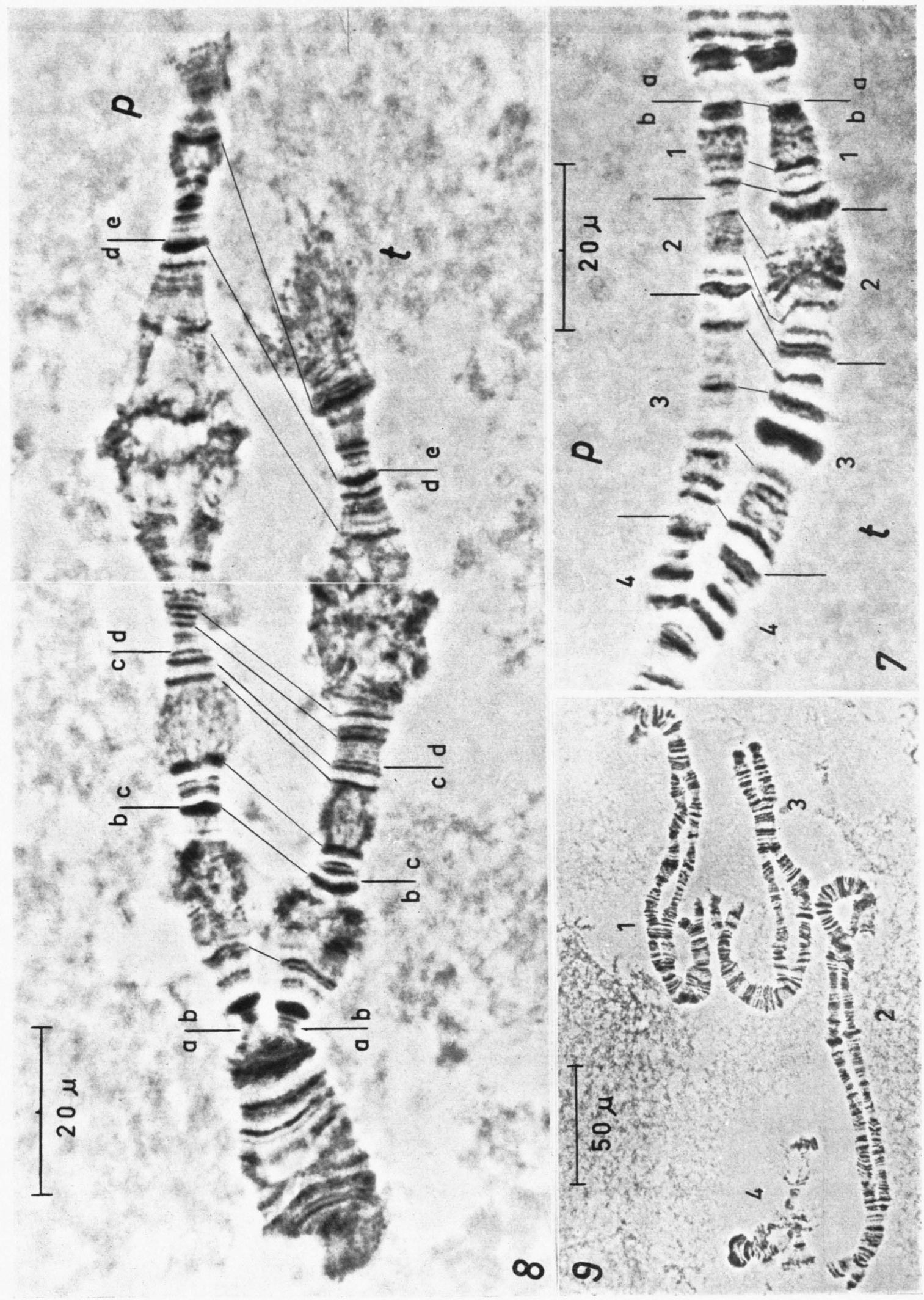

Abb. 7. Heterozygoter Mittelabschnitt von Chromosom III (Vergr. 1400-fach), 
Die Färbungsmerkmale, besonders die Intensität der Schwärzung der Mesonotalstreifen sowie die Form und Breite der abdominalen Querbinden können in gewissen Grenzen variieren. Die geringsten Schwankungen zeigen die Färbungsdifferenzen des Metanotums. Die Zuverlässigkeit dieses Merkmals konnte an Hand der Kreuzungsanalyse von 6 Freiland-Populationen gesichert werden.

Bei den Bastarden überwiegen in der $F_{1}$ im allgemeinen die Färbungscharaktere von piger. Die Mesonotalstreifen sind jedoch häufig stark verdunkelt wie bei thummi $(\hat{\jmath}, q)$; auch die abdominalen Querbinden sind,

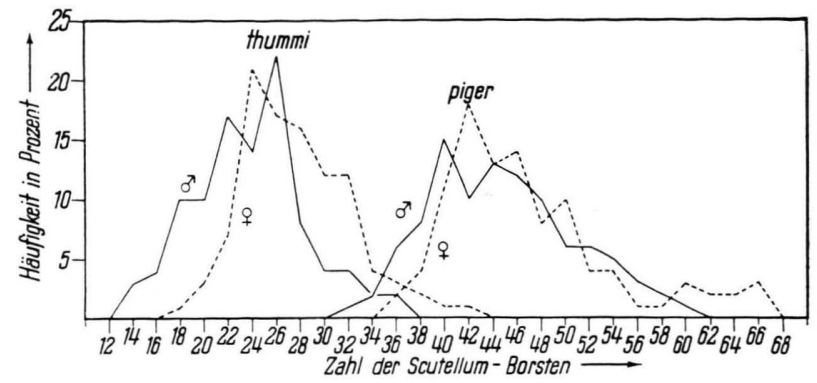

Abb. 2. Verteilung der Borstenzahlen des Scutellums bei den Imagines je einer Dauerzucht von Ch. th. thummi, Stamm $53(n=206 \hat{\jmath}$ o u. 138 oq $)$ und Ch.th. piger,

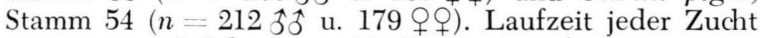
1 Jahr (etwa 19 Generationen).

besonders bei den $q Q$, gewöhnlich breiter. Auf dem Mesonotum kann die mediane Längslinie gut entwikkelt sein, ihre dreieckige Erweiterung vor dem Scutellum fehlt aber meist. Der Oralteil oder wenigstens der Vorderrand des Metanotums ist zwar oft, gewöhnlich flekkig oder wolkig, verdunkelt, doch hebt er sich stets von dem einheitlich schwarzbraun gefärbten Analteil deutlich ab. Bei zahlreichen Bastardindividuen, und zwar überwiegend $q \mathcal{q}$, ist die Verdunkelung des Oralteils des Metanotums sehr schwach oder gar nicht ausgeprägt, so daß eine sichere Unterscheidung von piger an Hand dieses Merkmals nicht möglich ist.

Außer der Färbung ließen die ersten Generationen der beiden ursprünglich isolierten Stämme von thummi und piger eine Reihe weiterer Unterschiede erkennen. Der größte Teil der in Tab. 1 erfaßten Merkmale zeigte aber entweder schon in den ersten (z. B. AR) oder in späteren Generationen (z. B. Zahl der Subapikalborsten des Stylus, Chätotaxie der Dorsalfläche des Analsegmentes, ferner die Form des Stylus und der Analspitze) eine so starke individuelle Variation, daß ihnen nur geringer Wert für die Differentialdiagnose zukommt. Die markantesten Unterschiede betreffen die Chätotaxie des Scutellums, die sich in den Dauerzuchten als verhältnismäßig konstant erwies (Abb. 2).

Wie in der Färbung zeigen die Bastarde von thummi und piger auch in diesem Merkmal starke Annäherung oder Angleichung an piger (Abb. 3).

Obwohl die beträchtlichen Differenzen in der Zahl der Scutellum-Borsten in den Dauerzuchten der einzelnen Stämme verhältnismäßig konstant blieben, erlauben auch sie keine durchgehende Trennung der beiden Rassen. Die Chätotaxie des Scutellums wird wie die Körpergröße (in den Tabellen ausgedrückt durch die Flügellänge) und die meisten anderen untersuchten Merkmale von den Milieuverhältnissen,

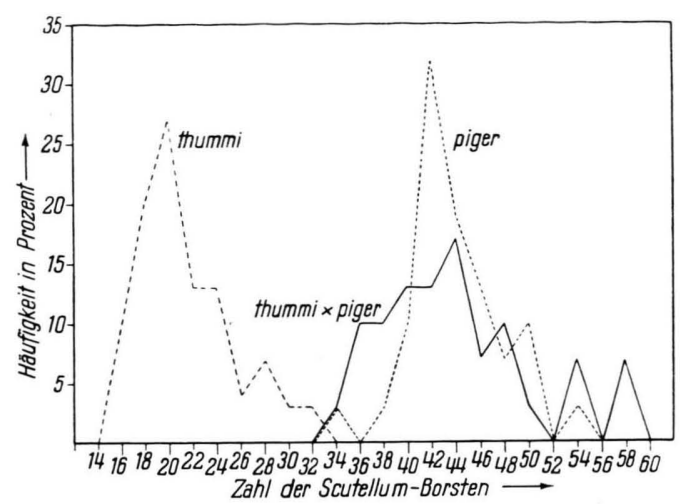

Abb. 3. Verteilung der Borstenzahlen des Scutellums bei den $\hat{\jmath} \hat{o}$ einer Kreuzung von Ch. th. thummi (Stamm 53) und Ch. th. piger (Stamm Dangast) $(n=$ je 30$)$.

unter denen die Larven aufwuchsen (besonders der Populationsdichte), beeinflußt. Tab. 2 gibt das Ergebnis von Versuchen wieder, in denen je 28, 70 und 280 aus einem Gelege stammende Larven in gleich großen Schalen (Bodenfläche etwa $280 \mathrm{~cm}^{2}$ ) mit der gleichen Gesamtmenge an Substrat und Futter aufgezogen wurden. Abgesehen von der z. T. erheblich verlängerten Larvalentwicklung trat bei beiden Unterarten in den mit 280 Larven besetzten Schalen mit der verringerten Körpergröße eine Verminderung der Borstenzahlen und des AR-Wertes ein. Die festgestellten Unterschiede machen es möglich, die in den einzelnen Zuchten beobachteten jahreszeitlichen Schwankungen der diagnostischen Werte zu erklären, zumal damit zu rechnen ist, daß Faktoren, die in den Versuchen möglichst optimal gehalten wurden (z. B. die $\mathrm{O}_{2}$-Versorgung), eine weitere Erhöhung der Differenzen verursachen können.

Das Ausmaß der von diesen Faktoren bestimmten Modifikation reicht jedoch nicht aus, um die Differenzen in der Chätotaxie zu erklären, die zwischen den Ausgangsstämmen und zahlreichen aus Freilandgelegen und -larven im Laboratorium aufgezogenen P-Generationen festgestellt wurden. Im allgemeinen weist piger zwar die für diese Unterart nach den 


\begin{tabular}{|c|c|c|c|c|}
\hline & thummi & $n$ & piger & $n$ \\
\hline Flügellänge & $3,1 \pm 0,26$ & 226 & $3,7 \pm 0,21$ & 138 \\
\hline Flügellänge & $3,1 \pm 0,28$ & 167 & $3,8 \pm 0.28$ & 136 \\
\hline $\begin{array}{l}\text { Zahl der Scutellum- } \\
\text { Borsten }\end{array}$ & $23,0 \pm 4,65$ & 236 & $47,3 \pm 5,58 \mid$ & 141 \\
\hline $\begin{array}{l}\text { Zahl der Scutellum- } \\
\text { Borsten }\end{array}$ & $27,5 \pm 4,48$ & 139 & $54,6 \pm 7,16$ & 135 \\
\hline $\begin{array}{l}\text { Zahl der Subapikal- } \\
\text { Borsten auf } \\
\text { dem Stylus }\end{array}$ & $8,7+0,89$ & 203 & $11,7 \pm 1,45$ & 162 \\
\hline $\begin{array}{l}\text { Zahl der Dorsal- } \\
\text { Borsten auf dem } \\
\text { Analsegment }\end{array}$ & $9,0 \pm 2,79$ & 203 & $14,9 \pm 4,54$ & 162 \\
\hline $\begin{array}{l}\text { Antennenverhältnis } \\
\quad(A R)\end{array}$ & $3,1-0,2$ & 124 & $3,5=0,17$ & 144 \\
\hline
\end{tabular}

Tab. 1. Mittelwerte und Standardabweichung der diagnostischen Merkmale von Chironomus thummi thummi und Chironomus thummi piger aus zwei monatlich untersuchten Dauerzuchten (Laufzeit $1 \mathrm{Jahr}=$ etwa 19 Generationen).

Stammzuchten charakteristischen höheren Werte auf; die Unterschiede gegenüber thummi sind jedoch, auch bei gleicher Körpergröße (Flügellänge), z. T. sehr gering. Vielfache Überschneidungen der Werte zeigen, daß außer der Färbung keine durchgehenden Unterscheidungsmerkmale erfaßt wurden (Tab. 3). Da die Werte in den auf Freilandgelegen basierten Dauer- und Einzelzuchten * innerhalb der zu erwartenden modifikatorisch bedingten Grenzen konstant blieben (Abb.4), sind die beobachteten Differenzen vermutlich auf genetische Unterschiede zwischen den einzelnen Freiland-Populationen der beiden Subspezies zurückzuführen.

\section{II.}

Eine sichere Unterscheidung der beiden Subspezies ist mit Hilfe der Struktur der Speicheldrüsen-Chromosomen möglich. Zur Kennzeichnung der Unterschiede eignen sich am besten die Chromosomen des Bastards, weil dort struktur-heterozygote Regionen durch Änderung des Paarungsbildes sofort auffallen; außerdem erlaubt die Ausbildung der Chromosomen beider Ausgangsformen unter gleichartigen Bedingungen eine unmittelbaren Vergleich**. Der Karyo-

* Dauerzuchten wurden in Kachelbecken mit geräumigen Flugkäfigen gehalten; abgesehen von wöchentlichen Futtergaben, Wasserwechsel in etwa 3-monatigem Abstand und laufender Abnahme des größten Teils der Gelege konnten sich die Folgegenerationen der auf einem Gelege basierenden P-Generationen in diesen Zuchten unter ständiger Panmixie entwickeln. In den Einzelzuchten wurden Einzelgelege in kleineren Becken aufgezogen; für die Weiterzucht wurde jeweils nur ein durch Geschwisterpaarung befruchtetes Gelege verwendet.

** Eine genaue Darstellung aller Struktur-Verschiedenheiten und die Veröffentlichung der Chromosomenkarten erfolgt an anderer Stelle. typ von Ch.thummi (B a u e r ${ }^{6}$ ) besteht aus 4 Chromosomen, von denen die mit I und II bezeichneten ungefähr gleich lang sind, das III. um ein Drittel kürzer ist, während die Länge des IV., welches 2 Balbianiringe und den Nukleolus enthält, nur ungefähr ein Viertel von der des Chromosoms I beträgt. Die Chromosomen I, II und III des Bastards thummi $\times$ piger sind in ihrem mittleren Teil und meist auch an den Enden ungepaart (Abb. 9***), das IV. Chromosom ist nur in einem Endabschnitt gepaart (Abb. 8, 9). Die Chromosomen der beiden Subspezies unterscheiden sich am auffälligsten durch je eine kräftige Ver-

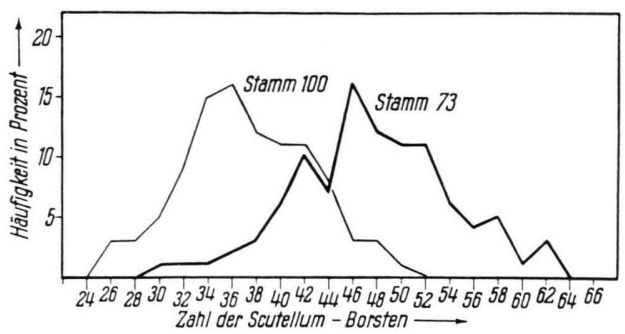

Abb. 4. Verteilung der Borstenzahlen des Scutellums bei den $\hat{\delta} \hat{o}$ zweier Dauerzuchten von $C h$.th. piger. Laufzeit 7 (Stamm 100, $n=80$ ) und 11 Monate (Stamm 73, $n=111)$.

dickung bei thummi, die ungefähr in der Mitte der medianen Paarungslücke der drei langen Chromosomen lokalisiert ist (Abb. 5, 6, 7); im Chromosom IV befindet sich eine derartige Aufblähung im Endabschnitt e (Abb. 8). Dagegen sind solche Anschwellungen bei piger in den Chromosomen I und II nur angedeutet (Abb. 5, 6) und fehlen im III. und IV. Chromosom völlig (Abb. 7, 8). B a u er ${ }^{6,7}$ deutet diese Regionen als heterochromatisch und als Sitz des Kinetochors, womit sich die drei langen Chromosomen als mediokinetisch und das IV. als telokinetisch erweisen $* * * *$.

Außer in der verschiedenen Ausbildung der Kinetochor-Region manifestiert sich die Heterozygotie durch unterschiedliche Färbung und Dicke einzelner Querscheiben, jedoch niemals durch Veränderung der Scheibenordnung (Inversionen). Diese Verhältnisse

6 H. B a u e r, Z. Zellforsch. mikroskop. Anatom. 23, 280 [1935].

*** Abb. 5-9 s. Tafel S. 728 a u. b.

7 H. B a u e r, Zool. Jb. Physiol. 56, 239 [1936].

**** Eigene Untersuchungen an Speicheldrüsen-Chromosomen von $F_{1}$-Nachkommen röntgenbestrahlter $\hat{\delta} \hat{\sigma}$ von Ch. thummi thummi bestätigen diese Deutung. Unter den reziproken Translokationen wurden niemals Fälle beobachtet, in denen zwei der angenommen KinetochorRegionen in einem Chromosom vereinigt sind. 


\begin{tabular}{|c|c|c|c|c|c|c|c|c|c|c|c|c|}
\hline \multirow{4}{*}{$\begin{array}{l}\text { Zahl der Larven } \\
\text { pro Schale } \\
\text { geschlüpfte } \\
\text { Imagines [ }[\%] \\
\left(\sigma^{7}, 9\right) \\
\text { Entwicklungsd. } \\
\text { (Larve+Puppe) } \\
\text { in Tagen }\end{array}$} & \multicolumn{6}{|c|}{ Ch. th. thummi (Stamm 53) } & \multicolumn{6}{|c|}{ Ch. th. piger (Stamm 100) } \\
\hline & \multicolumn{2}{|c|}{28} & \multicolumn{2}{|c|}{70} & \multicolumn{2}{|c|}{280} & \multicolumn{2}{|c|}{28} & \multicolumn{2}{|c|}{70} & \multicolumn{2}{|c|}{280} \\
\hline & \multicolumn{2}{|c|}{92,9} & \multicolumn{2}{|c|}{94,3} & \multicolumn{2}{|c|}{81,4} & \multicolumn{2}{|c|}{82,1} & \multicolumn{2}{|c|}{90,0} & \multicolumn{2}{|c|}{42,9} \\
\hline & \multicolumn{2}{|c|}{$17-24$} & \multicolumn{2}{|c|}{$18-24$} & \multicolumn{2}{|c|}{$11-49$} & \multicolumn{2}{|c|}{$28-22$} & \multicolumn{2}{|c|}{$18-24$} & \multicolumn{2}{|c|}{$20-56$} \\
\hline Flügellänge & $\begin{array}{c}3,3 \\
3,2-3,4\end{array}$ & $\begin{array}{c}9 \\
3,5 \\
3,4-3,6\end{array}$ & $\begin{array}{c}3 \\
3,4 \\
3,4-3,5\end{array}$ & $\begin{array}{c}3,8 \\
3,7-3,9\end{array}$ & $\left|\begin{array}{c} \\
2,7-3 \\
2,9\end{array}\right|$ & $\begin{array}{c}7 \\
2,9 \\
2,7-3,1\end{array}$ & $\mid \begin{array}{c}5 \\
3,8 \\
3,6-3,9\end{array}$ & $\begin{array}{c}q \\
4,1 \\
4,1-4,2\end{array}$ & $3,7-4,0$ & $\begin{array}{c}0 \\
4,0 \\
3,8-4,2\end{array}$ & $\begin{array}{c}3 \\
3,2 \\
3,0-3,4\end{array}$ & \begin{tabular}{l|c} 
& 9 \\
3,1 \\
$3,1-3,3$
\end{tabular} \\
\hline $\begin{array}{l}\text { Zahl der Scutel- } \\
\text { lum-Borsten }\end{array}$ & $\begin{array}{c}32,9 \\
25-41\end{array}$ & $\begin{array}{c}38,7 \\
37-42\end{array}$ & $\begin{array}{c}33,6 \\
29-38\end{array}$ & $\begin{array}{c}40,7 \\
34-49\end{array}$ & $25-31$ & $\begin{array}{c}32,8 \\
28-37\end{array}$ & $\begin{array}{c}40,4 \\
30-52\end{array}$ & $\begin{array}{c}60,0 \\
54-69\end{array}$ & $\begin{array}{c}39,3 \\
35-44\end{array}$ & $\begin{array}{c}54,2 \\
48-59\end{array}$ & $\begin{array}{c}36,4 \\
31-42\end{array}$ & $\begin{array}{c}40,1 \\
35-44\end{array}$ \\
\hline $\begin{array}{l}\text { Zahl der Sub- } \\
\text { apikal-Borst. } \\
\text { auf dem } \\
\text { Stylus }\left(\sigma^{\prime}\right)\end{array}$ & \multicolumn{2}{|c|}{$\begin{array}{c}9,7 \\
9-10\end{array}$} & \multicolumn{2}{|c|}{$\begin{array}{c}9,7 \\
9-10\end{array}$} & \multicolumn{2}{|c|}{$\begin{array}{c}9,2 \\
8-10\end{array}$} & \multicolumn{2}{|c|}{$\begin{array}{c}11,2 \\
10-12\end{array}$} & \multicolumn{2}{|c|}{$\begin{array}{c}10,9 \\
10-12\end{array}$} & \multicolumn{2}{|c|}{$\begin{array}{c}10,3 \\
10-12\end{array}$} \\
\hline $\begin{array}{l}\text { Zahl der Dorsal- } \\
\text { Borsten auf } \\
\text { dem Analseg- } \\
\text { ment }\end{array}$ & \multicolumn{2}{|c|}{$4-11$} & \multicolumn{2}{|c|}{$\begin{array}{c}7,6 \\
4-10\end{array}$} & \multicolumn{2}{|c|}{$\begin{array}{c}7,2 \\
2-10\end{array}$} & \multicolumn{2}{|c|}{$\begin{array}{c}16,1 \\
13-22\end{array}$} & \multicolumn{2}{|c|}{$\begin{array}{c}16,2 \\
10-19\end{array}$} & \multicolumn{2}{|c|}{$\begin{array}{c}14,2 \\
10-19\end{array}$} \\
\hline $\begin{array}{l}\text { Antennenver- } \\
\text { hältnis } \\
(A R)\left(\sigma^{\top}\right)\end{array}$ & \multicolumn{2}{|c|}{$\begin{array}{c}3,2 \\
3,0-3,3\end{array}$} & \multicolumn{2}{|c|}{$\begin{array}{c}3,2 \\
3,0-3,4\end{array}$} & \multicolumn{2}{|c|}{$\begin{array}{c}3,0 \\
2,8-3,3\end{array}$} & \multicolumn{2}{|c|}{$\begin{array}{c}3,4 \\
3,3-3,6\end{array}$} & \multicolumn{2}{|c|}{$\begin{array}{c}3,3 \\
3,2-3,6\end{array}$} & & $\begin{array}{l}3,1 \\
-3,3\end{array}$ \\
\hline
\end{tabular}

Tab. 2. Mittel- und Extremwerte der diagnostischen Merkmale von je 10 Imagines aus verschieden stark besetzten Zuchtschalen mit gleicher Gesamtmenge an Substrat und Futter.

lassen sich am einfachsten bei den Chromosomen I und IV erkennen. Dort erlaubt das Vorhandensein deutlicher Scheibengruppen eine leichte Identifizierung der homologen Chromosomen-Abschnitte.

Bei Chromosom I (Abb. 5) herrscht in den Abschnitten b 3, b 4 und e 2 völlige Übereinstimmung in allen Struktureinzelheiten; c 1, c 2, c 3, c 4, d 1 und e 1 unterscheiden sich lediglich durch die stärkere Färbung bzw. größere Dicke einzelner Scheiben bei thummi. Die Kinetochor-Region erweist sich für eine Analyse der Scheibenanordnung als besonders schwierig. Da aber der Grad der Aufblähung variabel ist, gelingt es, solche Fälle zu finden, die auch für diesen Abschnitt einen Vergleich mit der Scheibenfolge von piger zulassen.

In dem Chromosom IV (Abb. 8) sind außer der unterschiedlichen Gestalt der kinetochoren-tragenden Enden im Abschnitt e keine Anzeichen einer Heterozygotie in der Querscheiben-Ausbildung zu erkennen. Die zwischen den Balbianiringen und dem Nukleolus liegenden Scheibengruppen sind in allen Einzelheiten gleichartig. Bei der Variabilität der Gestalt der Balbianiringe und des Nukleolus-Bildungsortes ist es unmöglich, etwaige geringfügige Unterschiede dieser Strukturen festzustellen.

Die Schwierigkeiten, die bei der Homologisierung des heterozygoten Teils von Chromosom II (Abb. 6) entstehen, liegen allein in der dichten Aufeinanderfolge dünner, meist wenig markanter Scheiben. Mehr oder weniger große Färbungs- und geringe Abstandsunterschiede, die auf der Abbildung nicht zu erkennen sind, bilden die einzigen Unterscheidungsmerkmale zwischen den beiden Unterarten.

Der heterozygote Mittelteil von Chromosom III (Abb. 7) zeigt von allen 4 Chromosomen die erheblichsten Verschiedenheiten. An Stelle der mit mehreren stark gefärbten Scheiben ausgestatteten heterochromatischen Aufblähung in b 2 von thummi steht bei piger eine blasse Scheibengruppe. Zwei sehr dicke Scheiben bei thummi (b 1 und b 3) haben bei piger dünne, kaum sichtbare Homologe. Im übrigen herrscht Übereinstimmung in Gruppenbildung und Scheibenzahl.

Eine cytologische Rassendifferenzierung, die ausschließlich auf Unterschiede in der Querscheiben-Ausprägung und nicht auf Veränderungen der Genordnung innerhalb der heterozygoten Regionen beruht, ist bisher bei den Chironomiden noch nicht beschrieben worden*. E. Goldschmidt ${ }^{3}$ gibt für den

* Ein Beispiel einer offenbar ähnlichen cytologischen Differenzierung bildet der Bastard von Sciara ocellaris $\times$ S. reynoldsi (C. W. M e t z u. E. G. L a w r e n c e, J. Heredity 29, 176 [1938]). Die Speicheldrüsen-Chromosomen dieses Bastards sind inversionsfrei und zeigen in ihFortsetzung S. 732 


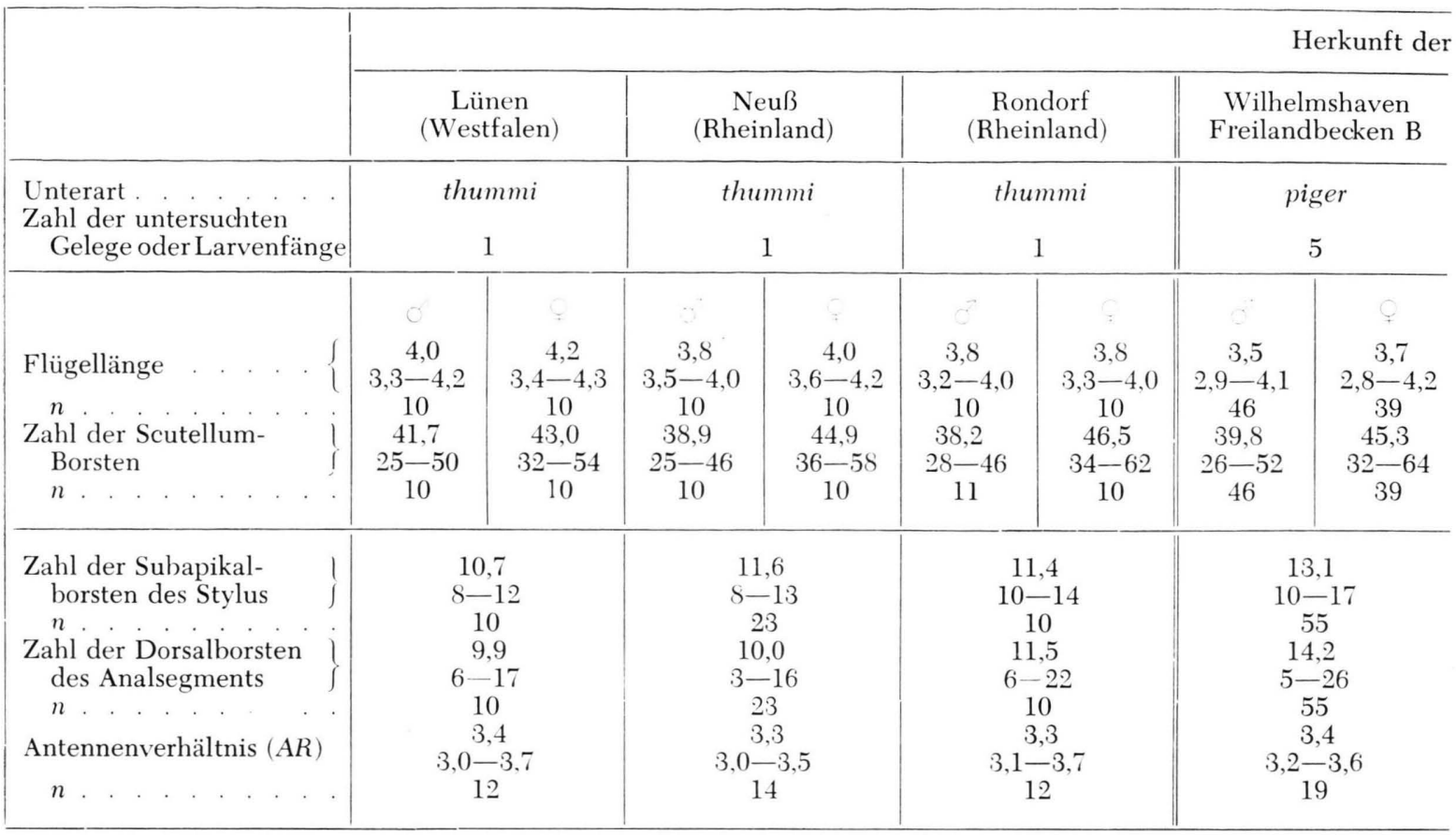

Tab. 3. Mittel- und Extremwerte der diagnostischen Merkmale

Bastard der zwei palästinensischen Formen von $C h$. thummi an, daß die Chromosomen in ihren ungepaarten Abschnitten eine nicht homologisierbare Querscheiben-Anordnung besitzen, und vermutet, daß die Differenzierung dieser Formen auf einer Umordnung kleiner Chromosomen-Abschnitte (minute rearrange' ments) beruhe* ${ }^{*}$ Eine derartige Deutung trifft für den Karyotyp unseres Bastards nicht zu. Die Differenzierung der Subspezies thummi und piger geschieht, soweit sich das cytologisch beurteilen läßt, offensichtlich durch Veränderungen in den Querscheiben selbst, die sich als quantitative Unterschiede der Färbbarkeit ausdrücken. Die Bedeutung, die ausgesprochenen Heterochromatin-Mutationen dabei zufällt, läßt sich nicht scharf abgrenzen, da es gegenwärtig nicht möglich ist, Heterochromatin streng zu definieren. Eine gewisse Wahrscheinlichkeit, daß außerhalb der Kinetochor-Region Heterochromatin an dem Differenzierungs-Prozeß beteiligt ist, besteht bei Chromosom III. Die beiden in den Abschnitten b 2 und b 3 des thummi-Chromosoms liegenden auf-

ren ungepaarten Regionen Unterschiede in der Färbung und Ausbildung einzelner Scheiben und Scheibengruppen. Ähnliche Erscheinungen treten auch bei den kleinen Strukturmutationen auf, die $\mathrm{B}$ e e r m a n ${ }^{2}$ neben hochgradiger Inversionsheterozygotie bei dem Artbastard Chironomus tentans $\times$ C. pallidivittatus gefunden hat.

* Aus den veröffentlichten Illustrationen ist ein strenger Beweis für diese Deutung nicht zu entnehmen. Bei fallend dicken Scheiben machen den Eindruck eingeschobener Heterochromatin-Blöcke. Dagegen dürften die weniger starken Färbungsunterschiede, wie sie vorwiegend im Chromosom II auftreten, auf Veränderungen im euchromatischen Material beruhen.

Das streckenweise Ausbleiben der Paarung in den Bastardchromosomen kann nicht die unmittelbare Folge der Struktur-Heterozygotie sein, da im Pachytän eine normale Paarung beobachtet worden ist*. Die Ursachen dieser Erscheinung, die auf physiologische Differenzen in den betreffenden ChromosomenAbschnitten der beiden Unterarten liegen dürften, sind unbekannt.

III.

Die Frage, ob außer den morphologischen und cytologischen auch bionomische, ethologische oder ökologische Unterschiede zwischen thummi und piger bestehen, war von besonderem Interesse. Bei etwa gleichen Eizahlen sind die Gelege von piger z. B. im allgemeinen bedeutend voluminöser als die von thummi, was offensichtlich auf einer stärkeren Quellbarkeit der gallertigen Grund-

der sonstigen Ähnlichkeit mit dem hier beschriebenen Bastard bezüglich der Anschwellungen und der Paarungslücken erscheint es nicht ausgeschlossen, daß die G ol d $\mathrm{s} \mathrm{ch} \mathrm{midtsche} \mathrm{Interpretation} \mathrm{unrichtig} \mathrm{ist.}$

* B a u e r mdl.

Nach unveröffentlichten Untersuchungen von Ito ist Chiasmabildung in den in Speicheldrüsen-Chromosomen ungepaarten Bereichen möglich, wie das Auftreten von crossing-over-Fällen in $F_{2}$ - und Rückkreuzungen beweist. 


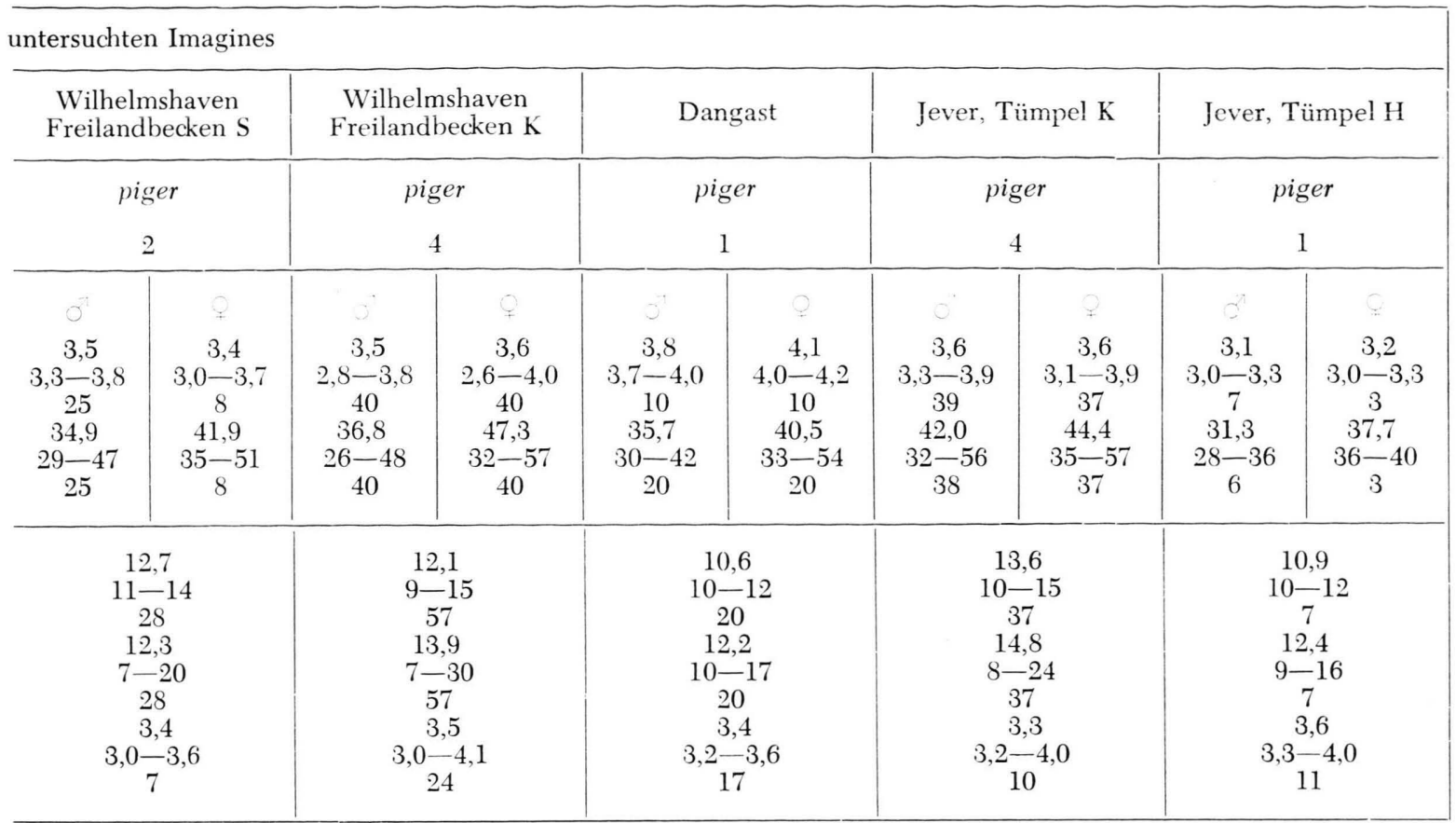

von 9 Freilandpopulationen von Chironomus thummi.

substanz beruht. Vermutlich ist dadurch auch die häufig zu beobachtende geringere Haftfähigkeit der Gelege am Substrat bedingt. Da aber sowohl die Zahl der Eier im Gelege wie die Wasseraufnahme der Gallerte von verschiedenen Außenfaktoren abhängen, ermöglicht dieses Merkmal keine durchgehende Trennung der beiden Subspezies. Unterschiede im Paarungsverhalten der Imagines, auf die Beobachtungen an den zuerst isolierten Stämmen hinzuweisen schienen, ließen sich trotz Variation der Größe und Form der Flugkäfige, der relativen Luftfeuchtigkeit, der Lichtintensität, der Luftbewegung und der Zahl der verwendeten kopulationsfähigen Imagines experimentell nicht nachweisen. Obwohl thummi im allgemeinen flugfreudiger als piger ist und auch in engräumigen Flugkäfigen (bis etwa $5 l$ Luftraum) einen höheren Prozentsatz an entwicklungsfähigen Gelegen liefert, hängen beide Erscheinungen wahrscheinlich nicht oder wenigstens nicht ausschließlich mit einer höheren Kopulations-Bereitschaft dieser Unterart unter LaboratoriumsVerhältnissen zusammen. Einzelzuchten aus Freilandgelegen zeigten, daß die erste Generation beider Subspezies in gleich großen Flugräumen annähernd gleiche Mengen an vollständig entwicklungsfähigen Gelegen hervorbringen kann. Erst bei der Weiterzucht der durch Geschwisterpaarung befruchteten Gelege machen sich gewisse Unterschiede bemerkbar. Trotz Konstanthaltung der Flugraumgröße und, soweit wie möglich, auch der physiographischen Außenfaktoren, nimmt die Zahl der entwicklungsfähigen Gelege bei piger gewöhnlich schon in den ersten Generationen sehr stark ab. Nach durchschnittlich 2 bis 3 Generationen (im Minimum in der $\mathrm{P}$, im Maximum in der $F_{s}$ ) werden keine entwicklungsfähigen Eier mehr hervorgebracht. Auch thummi zeigt bei Inzucht eine Ab- nahme der entwicklungsfähigen Gelege im Laufe der Generationen. Das Absinken der Fertilität erfolgt in den meisten Einzelzuchten dieser Rasse jedoch weniger abrupt als bei piger, und durchschnittlich werden zahlreichere Inzucht-Generationen durchlaufen (minimal 5, maximal 12), ehe keine entwicklungsfähigen Eier mehr abgelegt werden. An den Imagines der späteren Generationen mehrerer Inzuchtstämme traten zunehmend Mißbildungen auf (permanente Spreizstellung der Flügel, Reduktion der Antennen und der Vordertarsen), die wahrscheinlich mit einer Verringerung der Kopulationsfähigkeit und allgemein einer Herabsetzung der Vitalität verknüpft sind. Mit dem unterschiedlichen Verhalten der beiden Unterarten bei Inzucht erklärt sich vermutlich auch der im Mittel wesentlich höhere Anteil an total entwicklungsfähigen Gelegen in den Dauerzuchten von thummi. Von $4006 \mathrm{Ge}-$ legen einer über 12 Monate laufenden Dauerzucht dieser Subspezies waren $78,6 \%$ total, $18,4 \%$ partiell und $3,0 \%$ nicht entwickelt. 10676 Gelege aus 3 Dauerzuchten von piger setzten sich dagegen aus 59,6\% total, 22,7\% partiell und $17,7 \%$ nicht entwickelten Gelegen zusammen. Crowding-Effekte können sich auf diese Weise in den Zuchtbecken mit thummi stärker auswirken, was natürlich bei der Beurteilung der in den Dauerzuchten dieser Unterart zu beobachtenden geringeren durchschnittlichen Körpergröße und Borstenzahlen zu berücksichtigen ist.

Hinweise auf ein unterschiedliches ökologisches Verhalten der beiden Subspezies waren aus den Laboratoriums-Zuchten nicht zu gewinnen. Dagegen machen die vorliegenden Befunde eine verschiedene Verteilung auf die Freiland-Biotope wahrscheinlich. In der Umgebung von Wilhelmshaven wurden bis- 


\begin{tabular}{|c|c|c|c|c|c|c|c|c|c|c|}
\hline \multicolumn{2}{|c|}{\begin{tabular}{c|} 
Zahl der \\
verwendeten
\end{tabular}} & \multicolumn{9}{|c|}{ Zahl der Gelege mit $\%$ entwickelten Eiern } \\
\hline$\sigma^{2}$ & Qq & $\Sigma$ & 100 & $>90$ & $>75$ & $>50$ & $>25$ & $>10$ & $|<10|$ & 0 \\
\hline \multicolumn{11}{|c|}{ a. thummi $\times$ thummi } \\
\hline 44 & 30 & 30 & 18 & 9 & & & 1 & & & 2 \\
\hline 28 & 25 & 20 & 11 & 2 & & & 2 & & & 5 \\
\hline 69 & 12 & 12 & 2 & 5 & 1 & 1 & & & & 3 \\
\hline 141 & 67 & $\begin{array}{r}62 \\
100\end{array}$ & $\begin{array}{l}31 \\
50\end{array}$ & $\begin{array}{l}16 \\
25,8\end{array}$ & $\begin{array}{l}1 \\
1,6\end{array}$ & $\begin{array}{l}1 \\
1,6\end{array}$ & $\begin{array}{l}3 \\
4,9\end{array}$ & & & $\begin{array}{l}10 \\
16,1 \%\end{array}$ \\
\hline \multicolumn{11}{|c|}{ b. piger $\times$ piger } \\
\hline 122 & 85 & 71 & 20 & 10 & 1 & 1 & & & & 39 \\
\hline 70 & 20 & 17 & 9 & & & 1 & & 1 & & 6 \\
\hline 57 & 18 & 15 & & 2 & & & & & & 13 \\
\hline 249 & 123 & 103 & 29 & 12 & 1 & 2 & & 1 & & \\
\hline & & 100 & 28,1 & 11,7 & 1,0 & 1,9 & & 1,0 & & $56,3 \%$ \\
\hline \multicolumn{11}{|c|}{ c. thummi $\times$ piger } \\
\hline 150 & 76 & 68 & 1 & 2 & & 3 & 1 & 2 & 13 & 46 \\
\hline 70 & 18 & 15 & & & 1 & & & 1 & 2 & 11 \\
\hline 70 & 23 & 12 & & & & & & & 1 & 11 \\
\hline 50 & 27 & 23 & 4 & 5 & 1 & 1 & 1 & & & 11 \\
\hline 39 & 30 & 10 & 4 & 1 & & & & & & 5 \\
\hline 42 & 16 & 6 & & & & 1 & & & & 5 \\
\hline 421 & 190 & 134 & 9 & 8 & 2 & 5 & 2 & 3 & 16 & \\
\hline & & 100 & 6,7 & 6,0 & 1,5 & 3,7 & 1,5 & 2,2 & 12,0 & $66,4^{\circ} / 0$ \\
\hline
\end{tabular}

Tab. 4. Entwicklungsfähigkeit der in 6 Kreuzungsversuchen erhaltenen Gelege.

her 9 Freiland-Populationen von Chironomus thummi in mehr oder weniger regelmäßigen Zeitabständen untersucht. Auf Grund der Färbungscharaktere, deren Zuverlässigkeit in 3 Fällen durch Kreuzung mit Laboratoriums-Stämmen und cytologische Analyse der Bastarde bestätigt werden konnte, handelte es sich ausnahmslos um reine Populationen von piger. Die Biotope, aus denen die Gelege oder Larven stammten, waren Kleingewässer in der Nähe von Bauerngehöften und auf Viehweiden sowie Versuchsbecken, deren Wasser durch Zugabe von Kuh- und Taubendünger eutrophiert worden war. Zu piger sind ferner Imagines zu stellen, die uns vom Ufer des Neusiedler Sees (Österreich) vorliegen (leg. M a i n x ,in einem halbversunkenen Boot") sowie die Populationen, die von $\mathrm{Th}$ i en e mann und $\mathrm{S} \operatorname{trenzke}{ }^{5}$ aus der Umgebung von Plön/Holstein (Gartenbecken, Dorfteich Hassberg) untersucht worden waren. Das Habitat dieser Unterart bildeten also in allen Fällen nichtperiodische, meist makrophyten-arme oder -freie Kleingewässer, die zwar (meist durch animalische Exkremente) hoch eutroph waren, aber keinen ausgeprägten Abwasser-Charakter hatten. In den Versuchsbecken vermochte sich piger bei Salinitätswerten bis $5,3 \%$ zu entwickeln.
Reine Populationen von Ch.th.thummi wurden im Wilhelmshavener Untersuchungsgebiet bisher nicht gefunden. Dagegen gehö̈rten die Larven, die wir von 3 Firmen als Aquarienfisch-Futter erhielten, entweder ausschließlich (Lünen/Westf., Neuss/Rheinl.) oder überwiegend (Rhondorf/Rheinland, hier etwa $10 \%$ Bastarde mit ungepaarten Chromosomen) thummi an. In allen Fällen wurde die Bestimmung durch Kreuzung mit Laborstämmen und cytologische Untersuchung der Bastarde gesichert. Die von $\mathrm{B}$ a u e ${ }^{6}$ untersuchten Larven, die von einer thüringischen Firma bezogen wurden, gehören auf Grund des Chromosomen-Baues ebenfalls zu thummi. Nach der Färbung müssen ferner sämtliche in der Sammlung Thienemanns enthaltenen Imagines aus dem Rheinland und Westfalen zu dieser Subspezies gestellt werden. Die Larven, aus denen sie gezüchtet wurden, stammen aus Gewässern, die durch das Einleiten organischer Abwässer hochgradig verunreinigt waren; sie werden nach $\mathrm{Thienemann}{ }^{8}$ von Kolkwitz und Marsson als typisch für die $\alpha$ mesosaprobe Zone bezeichnet, kommen aber auch noch in der poly- und $\beta$-mesosaproben Zone vor. Da

8 A. Thienemann, Chironomus. Stuttgart 1954. 
die als Fischfutter zu erhaltenden Larven gewöhnlich in Gewässern gesammelt werden, die einen ähnlichen limnologischen Charakter haben wie die von $\mathrm{Th}$ i e n e m a n n gekennzeichneten Abwasser-Fundstellen, scheint thummi demnach enger an saprobe Verhältnisse gebunden zu sein als piger.

Die vorliegenden Befunde machen es also wahrscheinlich, daß die beiden morphologisch und cytologisch differenzierbaren Unterarten von Chironomus thummi verschiedene ökologische Nischen einnehmen; piger bevorzugt die von $\mathrm{T} h$ i e $\mathrm{n}$ e $\mathrm{m}$ a $\mathrm{n} \mathrm{n}$ genannten eutrophen Kleingewässer und Seen, während thummi eine ausgeprägt saprophile, vielleicht sogar saprobionte Form wäre. Durch weitere Untersuchungen soll versucht werden, diese Auffassung zu bestätigen und die Milieuansprüche der Unterarten schärfer zu fassen. Eine geographische Trennung des heutigen Areals von thummi und piger liegt offensichtlich nicht vor (Auftreten von Bastarden in Wilhelmshaven und Rondorf). Rückschlüsse auf den Entstehungsmodus der intraspezifischen Differenzierung innerhalb der Art Chironomus thummi können aus den vorliegenden Funden nicht gezogen werden.

Bis zu welchem Grad die beiden Subspezies im Freiland reproduktiv isoliert sind, läßt sich noch nicht entscheiden. Im Versuch ist die wechselseitige Kopulation leicht herbeizuführen; allerdings ist der Anteil an nicht oder nur zu einem geringen Prozentsatz entwicklungsfähigen Gelegen bei der Paarung von $\hat{\jmath} \hat{\jmath}$ und $q Q$ verschiedener Unterarten größer als bei der Kreuzung von Angehörigen der gleichen Unterart (Tab. 4). Die Entwicklung der Bastard-Larven verläuft in der $F_{1}$ normal, und die Zahl der von den Bastard-Imagines der $F_{1}$ produzierten entwicklungsfähigen Gelege entspricht in Übereinstimmung mit den Ergebnissen Goldschmidts ${ }^{3}$ den Verhältnissen in homozygoten Populationen. Schon die Gesamtzahlen der bei den Kreuzungsversuchen erhaltenen Gelege deuten auf eine höhere Kopulationsrate zwischen Partnern der gleichen Unterart hin (Tab. 4); wegen des unterschiedlichen Alters der verwendeten $\hat{\jmath} \widehat{\jmath}$ können die Versuche in dieser Hinsicht aber nur mit Vorbehalt ausgewertet werden. In Flugkäfigen, in denen zu $\$+$ von thummi die gleiche Anzahl gleichaltriger $\hat{\jmath} \hat{\jmath}$ beider Subspezies gesetzt wurden, ließ sich eine ausgeprägte selektive Kopulations-Bereitschaft nachweisen. Es fanden ausschließlich oder überwiegend (85-100\%) Gleichpaarungen statt (cytologische Untersuchung der $\mathrm{F}_{1}$-Larven). Fremdpaarungen erfolgten nur am Ende der Versuche, als das ursprüngliche Zahlenverhältnis der $\hat{\delta} \hat{\jmath}$ von thummi und piger durch den Tod zahlreicher Imagines zufällige Verschiebungen erfahren hatte. Daß auch im Freiland die Kopulation zwischen Angehörigen verschiedener Subspezies möglich ist, zeigt die LarvenPopulation Rondorf, die etwa 10\% Heterozygote enthielt. Die Klärung der Frage, unter welchen Verhältnissen es im Freiland zur Bastardierung kommt und wie die Bastarde ökologisch eingestellt sind, muß weiteren Untersuchungen vorbehalten bleiben.

\title{
BERICHTE
}

\section{Die Hybridisierung der Atome und ihre Beziehungen zu Valenz und Struktur}

\section{Von GÜNTHER SCHOtT ${ }^{1}$}

\author{
Aus dem Institut für Anorganische Chemie der Universität Rostock \\ (Z. Naturforschg. 11 b, 735-747 [1956]; eingegangen am 20. Februar 1956)
}

$\mathrm{D}$ ie Konzeption des Hybridbegriffes hat sich seit seiner Einführung durch $\mathrm{Pauling}{ }^{2}$ als Arbeitshypothese so überaus bewährt und als derart leistungsfähig erwiesen, daß er ein nahezu unentbehrliches Hilfsmittel der theoretischen Chemie geworden ist. Nach Ansicht des Verfassers kommt ihm bei der Betrachtung struktureller Probleme eine absolut zentrale Bedeutung zu, da mit ihm alle experi-

1 Nach einem Vortrag anläßlich der Hauptjahrestagung 1955 der Chemischen Gesellschaft in der DDR in Leipzig am 20.10. 1955. mentell prüfbaren Strukturelemente, wie Bindungslänge, Bindungsenergie, Valenzwinkel, Dipolmoment usw., ursächlich und direkt verknüpft sind. Der Hybridbegriff verdient es also, auch dem mehr praktisch orientierten Chemiker nahegebracht zu werden. Aufgabe des Aufsatzes soll es sein, in diesem Sinne zwischen Theorie und Praxis zu vermitteln, den Hybridbegriff anschaulich zu erläutern und seine vielfältige Leistungsfähigkeit für Fragen der Valenz und Struktur darzulegen.

2 L. P a u ling, J. Amer. chem. Soc. 53, 1367 [1931]. 